\title{
Implementation of Algorithms For Multi-Channel Digital Monitoring Receiver
}

\author{
K Sridhar reddy, M.Tech(ES) $)^{1}$, Satish Kumar V, M.Tech(DE\&CS) ${ }^{2}$, K Satish \\ Reddy, MS(ECE) $)^{3}$, Shaik Basha, M.Tech(DE\&CS $)^{4}$. \\ (1.Associate professor in Vardhaman College of Engg., Hyderabad) \\ (2.Assistant Professor in Vignan's Institute of Tech. and Aeronautical Engg., Hyderabad) \\ (3.Associate professor in Vardhaman College of Engg., Hyderabad) \\ (4.Associate professor in Vardhaman College of Engg., Hyderabad).
}

\begin{abstract}
Monitoring Receivers form an important constituent of the Electronic support. In Monitoring Receiver we can monitor, demodulate or scan the multiple channels.

In this project, the Implementation of algorithm for multi channel digital monitoring receiver. The implementation will carry out the channelization by the way of Digital down Converters (DDCs) and Digital Base band Demodulation. The Intermediate Frequency (IF) at $10.7 \mathrm{MHz}$ will be digitalized using Analog to Digital Converter $(A D C)$ with sampling frequency $52.5 \mathrm{MHz}$ and further converted to Base band using DDCs. Virtually all the digital receivers perform channel access using a DDC. The Base band data will be streamed to the appropriate demodulators. Matlab Simulink will be used to simulate the logic modules before the implementation. This system will be prototyped on an FPGA based COTS (Commercial-off-the-shelf) development board. Xilinx System Generator will be used for the implementation of the algorithms.
\end{abstract}

Keywords: DDC, ADC, Digital Base band demodulation, IF, Monitoring Receiver.

\section{Introduction}

The emergence of a promising, versatile technology into the commercial world seems to set the entire communication industry into pure excitement. Not only has it definitely become a major focus of attention but it is also catalyzing enhancement of new standards as the industry is taking its big steps towards the age of "fifth generation" $5 \mathrm{G}$ communication. In general this project mainly serves two critical goals. Firstly, there was an interest in the area of development of a radio system based on utilization of programmable processing to enable the emulation of different military radio signals operating within a wide range of frequency. The other goal was targeted at creating the possibility of allowing future incorporation of new coding and demodulation standards in radio systems with advancement of new technologies. Various standardizations have been proposed to bring the radio frequency to the intermediate frequency at which the signal processing is easy and down converted to base-band signal. The base-band data is routed to the different analog demodulation schemes.

Monitoring Receiver forms an important constituent of Electronic Support (ES).

\section{Software Defined Radio}

Software-Defined Radio (SDR) is a rapidly evolving technology that is receiving enormous recognition and generating widespread interest in the telecommunication industry. Over the last few years, analog radio systems are being widely used for various radio applications in military, civilian and commercial spaces. In addition to this, programmable hardware modules are increasingly being used in analog radio systems at different functional levels. SDR technology aims to take advantage of these programmable hardware modules to build open architecture based radio system software. SDR technology facilitates implementation of some of the functional modules in a radio system such as modulation/demodulation, signal generation. This helps in building reconfigurable software radio systems where dynamic selection of parameters for each of the above-mentioned functional modules is possible. The main aim is to develop a model of Software defined Radio using SIMULINK tool. Software Defined Radio is a radio communications transceiver system in which all the typical components of a communication system such as mixers, modulators, demodulators, detectors are implemented through software rather than hardware.

The Objectives and goals of SDR are stated as follows:

- To develop a model of a Software Defined Radio which support the IEEE 802.11 a standard and Bluetooth standard using the SIMULINK tool.

- To implement all the main protocol stacks for WLAN and Bluetooth Protocols and verify their functionality.

- To implement point to point communication and data transfer for the Bluetooth standard. 


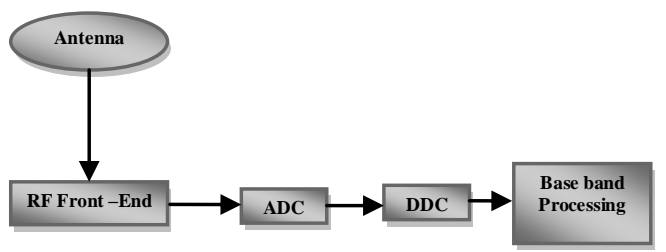

Fig:1. SDR Receiver

RF and IF Section : The RF section consists of essentially analog hardware modules while IF and base band sections contain hardware modules. The RF section is also called as RF front-end.

The RF section is responsible for transmitting/receiving the radiofrequency (RF) signal from the antenna via a coupler and converting the RF signal to an intermediate frequency (IF) signal. The RF front-end on the receive path performs RF amplification and analog down conversion from RF to IF. On the transmit path, $\mathrm{RF}$ front-end performs analog up conversion and RF power amplification.

ADC: The ADC blocks perform analog-to-digital conversion (on receive path) conversion. ADC blocks interface between the analog and digital sections of the radio system. DDC blocks perform digital-down conversion (on receive path).

DDC : The DDC/DUC and base band processing operations require large computing power and these modules are generally implemented using ASICs or stock DSPs. Implementation of the digital sections using ASICs results in fixed-function digital radio systems.

If DSPs are used for base band processing, a programmable digital radio (PDR) system can be realized. In other words, in a PDR system base band operations and link layer protocols are implemented in software.

The DDC functionality in a PDR system is implemented using ASICs. The limitation of this system is that any change made to the RF section of the system will impact the DDC operations and will require nontrivial changes to be made in DDC ASICs.

Base Band Processing:The base band section performs base band operations such as connection setup, equalization, frequency hopping, timing recovery; correlation and also implements the various demodulation schemes in order to recover the original signal which has been transmitted.

\section{Proposed System}

The system proposed can be simulated in two different approaches and the results of both the approaches at last point out the same outputs but according vary only in terms of the representation of the blocks which are used. The two approaches are

1. Using MATLAB Simulink Block Sets

2. Using MATLAB Simulink Xilinx Block Sets.

The systematic approach of both the methods will be discussed for the below mentioned block diagram.
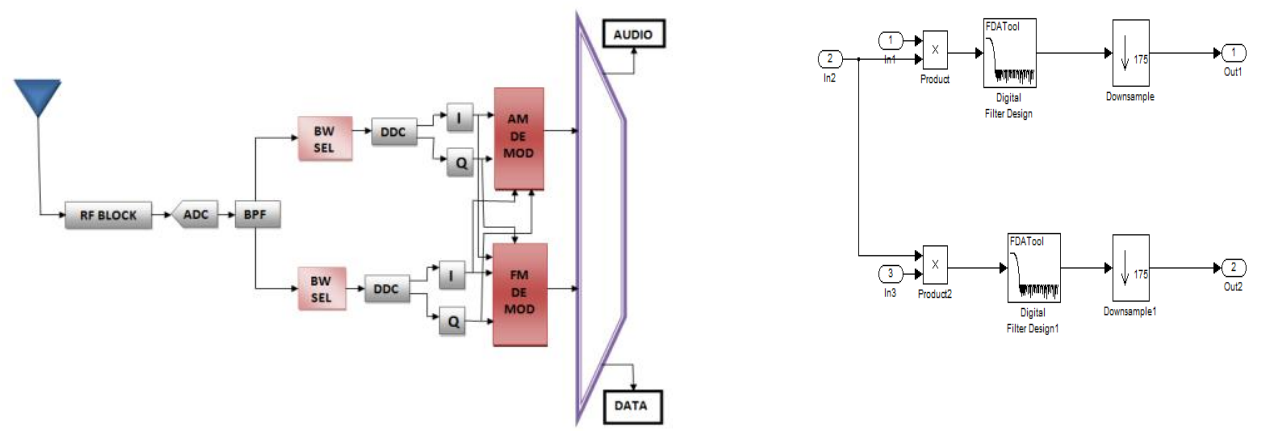

Fig:2. Block Diagram of proposed for Multi-channel Monitoring Receiver, Fig: 3 DDC of Single Channel

RF: This block mainly receives the signal from the antenna which ranges from $20-3000 \mathrm{MHz}$ of frequency and the bandwidth of the signal varies according to the signal type which is transmitted from the transmitter and received by the receiver.

Example: For FM, BW is $150 \mathrm{KHz}$.

The RF range is very high where the processing of the signal is difficult so it brought down to a frequency range known as "intermediate frequency (IF)" which is about $10.7 \mathrm{MHz}$. In simulation process, IF is directly taken as the input.

ADC: In MATLAB Simulink, this block is available as "Quantizer". The quantizer in this system proposed behaves as an analog to digital convertor. The symbol and its parameter setting play a vital role for it to be working like an ADC. In this ADC used will be 14 bit. Out of 14 bits 13 bits are used as the binary digits and rest 1 bit is used as either signed or unsigned bit. 
Digital Down Conveter(DDC): In the MATLAB SIMULINK, DDC is implemented using the product blocks whose output is given to the filter and with the help of downsampler the sample time is reduced to the desired range from 52.5MHz.The output of the DDC will be two components. They are

- In phase component (I)

- Quadrature phase component (Q).

The in phase component is obtained when same signals are multiplied with each other and quadrature phase component occurs when different signals are product with each other.DDC is mainly used for down converting the sample time from higher frequency to lower range where the processing is easy.

Band-Pass Filter (BPF): A Band-Pass Filter is a device that passes frequencies within a certain range and rejects (attenuates) frequencies outside that range. Band-pass is an adjective that describes a type of filter or filtering process. It is frequently confused with pass band, which refers to the actual portion of affected spectrum. A band-pass signal is a signal containing a band of frequencies away from zero frequency, such as a signal that comes out of a band-pass filter. An example of an analogue electronic band-pass filter is an RLC circuit. These filters can also be created by combining a low-pass filter with a high-pass filter.

\section{Implementation Of DDC}

This is the implementation of the digital down covertor using matlab simulink. Here, the input to two product multipliers are given two inputs one is the output of the ADC and other is sine and cosine signals to get the outputs I and Q components. The sample time through out the process must be same for any implementation in MATLAB.Here before it is down sampled to the sample frequency of $52.5 \mathrm{MHz}$ and after the down sampled the sample time is down converted to $300 \mathrm{KHz}$ where the signal processing is very easy.

The process how the DDC of down sample factor 175 is obtained is represented as in the below. The down sample factor 175 is multiplied using three factors 5,7,5 with filter at each stage.
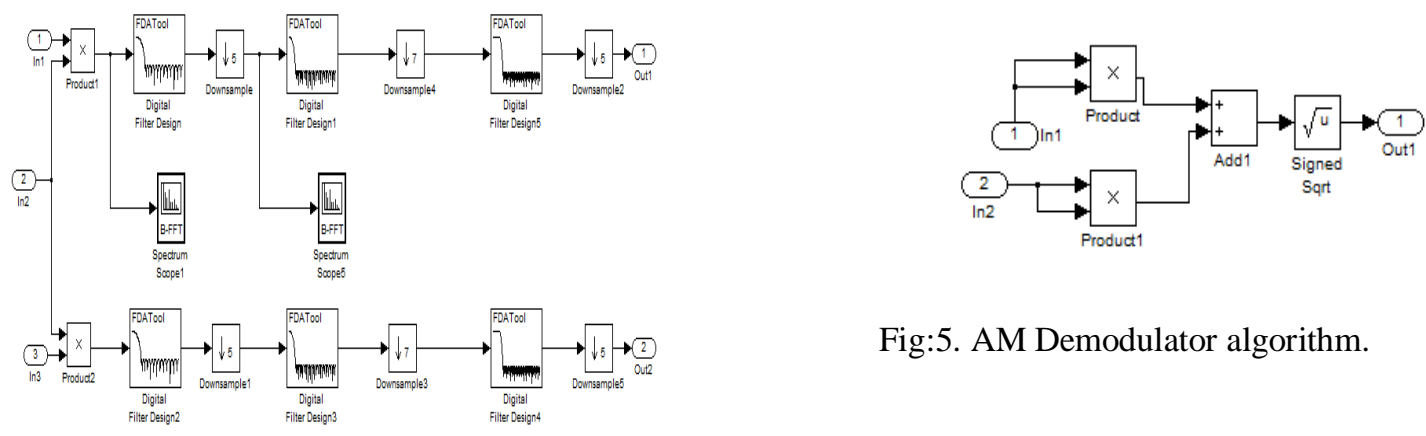

Fig:5. AM Demodulator algorithm.

Fig:4. DDC with different stages to form a down sample factor of 175 .
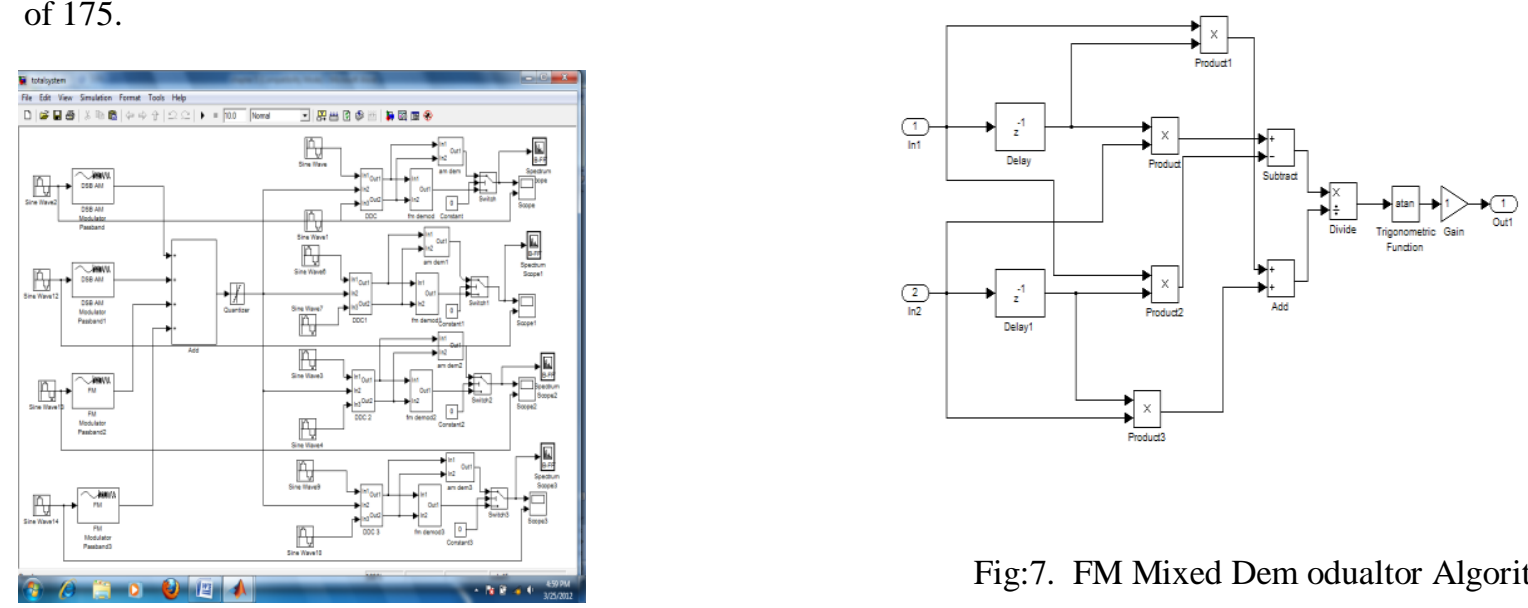

Fig:6.Implementation of Multi-channel Monitoring Receiver

The total implementation of the proposed system is represented further. As the signal which is received may be either AM or FM signals with the different carrier frequencies and have a sampling frequency of 
$52.5 \mathrm{MHz}$ where the signal processing is difficult so it is down sampled with a factor of 175 so the sampling frequency reduces to $300 \mathrm{KHz}$ of inphase and quadrature phase components which are given to the AM and FM analog demodulator schemes. The demodulator schemes are implemented using the below two demodulator techniques of AM and FM in practical as they differ from other schemes .

Based upon the output of DDC the inphase and quadrature phase components are given as the input to AM and FM demodulator.The output of the AM and FM are given to the switch with either high (1) or low (0) selection value to kown the desired signal.

\section{Results}

The output of the DDC depending upon the signal transmitted can be seen in spectrum scope which gives the frequency domain analysis of the signal where as the scope gives the time domain analysis of the signal.

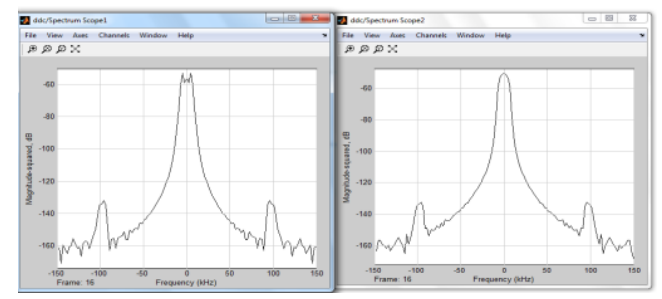

Fig:8. Frequency domain analysis of Inphase and Quadarture phase of DDC

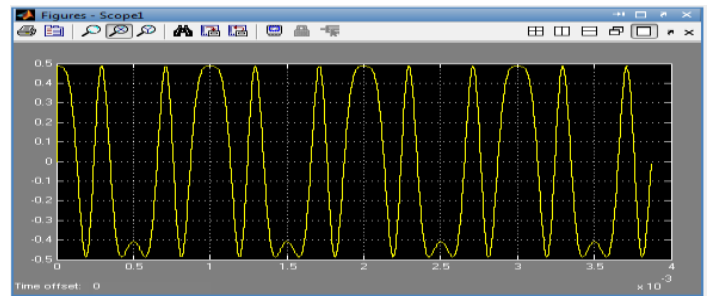

Fig:9.Time domain analysis of Quadrature component

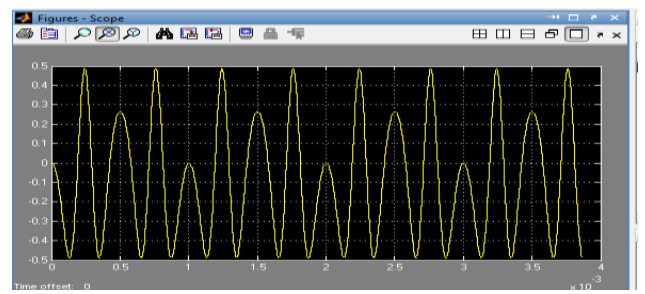

Fig:10.Time domain analysis of Inphase component of DDC

The results of the DDC will have the low sampling time of around $300 \mathrm{KHz}$ as the transmitted signal will have the high frequency of $52.5 \mathrm{MHz}$, where the signal can't be processed and the recovery of the orignial signal which is transmitted.

At the $52.5 \mathrm{MHz}$ the signal cannot be analised so it is down converted to $300 \mathrm{KHz}$ using a

down sampler factor of 175. Based upon the signal received from the antenna which is transmitted from transmitter which has a high sampling frequency of $52.5 \mathrm{MHz}$ which is down sampled to $300 \mathrm{KHz}$ with the help of DDC. Based upon the number of channels of different frequencies which are transmitted from transmitter which may be either AM or FM signals which are recovered after the DDC which may be having multiple DDC's whose outputs are inphase and quadrature phase components which are given to the suitable analog demodualtor techniques to recover the original signal which is transmitted.As the system which is proposed has been designed for receiver and recover of the signal which may be either AM or FM. The signal received by the antenna may be detected with the help of the switch and the signal of the desired is obtained from multiple signals of multi channels.

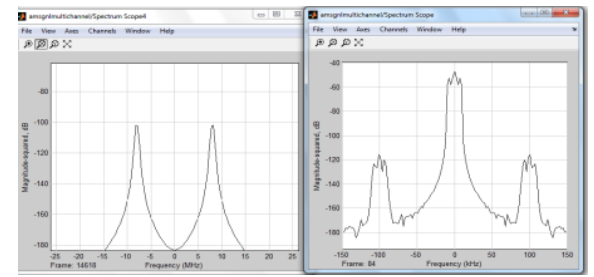

Fig:11. Frequency analysis of recovery of original $7 \mathrm{KHz}$ signal

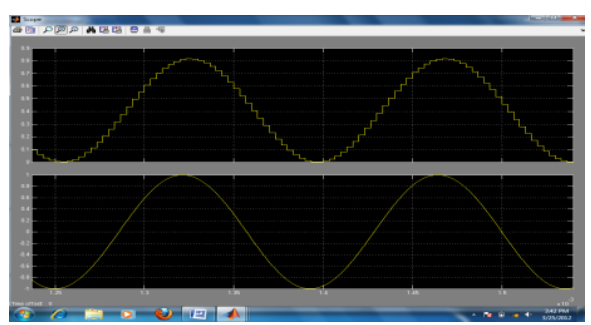

Fig:12. Time domain analysis of AM 7KHz signal

The above results implemented for the AM signal received by the receiver antenna for various carrier frequencies of $8,9,10,11 \mathrm{MHz}$ of sampling frequency $52.5 \mathrm{MHz}$ and using the DDC algorithm. The original signal 
has been recovered with the same carrier frequencies of the signal i.e. $8,9,10,11 \mathrm{MHz}$ of sampling frequency $300 \mathrm{KHz}$.The results are represented below with frequency and time domain analysis.

\section{Conclusion}

Finally we conclude that "IMPLEMENATTION OF ALGORITHMS FOR DIGITAL MONITORING RECEIVER" has an emerging application in the Military applications in determining the modern battle scenario protecting one's own forces from attack; deny information to the enemy and intercepting and disrupting an enemy's voice communication and data links.

The "IMPLEMENATTION OF ALGORITHMS FOR DIGITAL MONITORING RECEIVER" has been implemented using DDC'S for multi channels and the demodulation techniques used are only analog. As the now-a-days technology is tending towards digital techniques, we can implement this proposed system for digital demodulation techniques such as PSK, FSK, QPSK, ASK etc.

This proposed system may be of audio or data format and the signal processing and analysis of the signal can be extended to digital techniques too. The above proposed system can also be implement in Xilinx Block sets.

\section{References}

[1] http://www.sdrforum.org/faq.html

[2] http://www.xilinx.com/products/virtex4/overview/performance.htm.

[3] http://www.xilinx.com/xlnx/xil prodcatproduct.jsp?title=v2p_powerpc

[4] Virtex-4 Configuration Guide, Xilinx Inc, p. 81, 2004,http://direct.xilinx.com/bvdocs/userguides/ug071.pdf

[5] http://www.ep.liu.se/exjobb/isy/2003/3448/exjobb.pdf

[6] Murty, Ravi. (n.d.). Software-Defined Reconfigurable Radios: Smart, Agile, Cognitive, and Interoperable. Technology @Intel

[7] Magazine. Retrieved on November 3, 2004, from http://www.intel.com/update/contents/wi07031.htm

[8] Herbrig, H., Lundheim, L., Rossing, N. K., "SORT SW-Radio - From Concept towards Demonstration", Proceedings of the ACTS

Mobile Communications Summit 1999.

\section{AUTHOR BIOGRAPHIES}

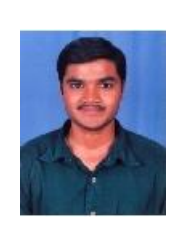

I Satish Kumar V, graduated from the Department of ECE in Joginpally B.R. Engg. college during 2008 at Hyderabad, M.TECH(DECS) from Sri Indu College of Engineering. \& Technology and published two International conferences and National Conference and interested Research in Image Processing and communication.

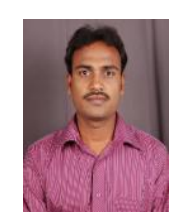

I Sridhar Reddy K, graduated from the Department of ECE in Farah College of Engg. \&Tech in 2005 at Hyderabad, M.TECH(EMBEDDED SYSTEMS) from CMR College of Engineering \&Technology and published two international Conference and interested Research in communication.

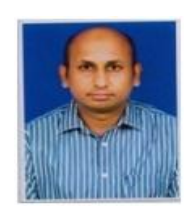

I K Satish Reddy graduated from the Department of ECE in JNTU, Hyderabad in 2001, MS(ECE) in USA and published one Naational Conference and interested in communication.

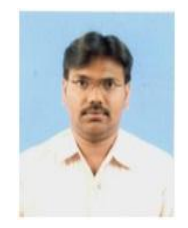

I Shaik Basha graduated from the Department of ECE in JNTU, Hyderabad in 2005, M.TECH(DECS) in JNTU, Kakinada and published two National Conferences and interested in Image processing and communication. 